\title{
Fuzzy Rule Based Multiwavelet ECG Signal Denoising
}

\author{
Bingo Wing-Kuen Ling, Charlotte Yuk-Fan Ho, Hak-Keung Lam, Thomas Pak-Lin Wong, Albert Yick- \\ Po Chan and Peter Kwong Shun Tam
}

\begin{abstract}
Since different multiwavelets, pre- and postfilters have different impulse responses and frequency responses, different multiwavelets, pre- and post-filters should be selected and applied at different noise levels for signal denoising if signals are corrupted by additive white Gaussian noises. In this paper, some fuzzy rules are formulated for integrating different multiwavelets, pre- and post-filters together so that expert knowledge on employing different multiwavelets, pre- and post-filters at different noise levels on denoising performances is exploited. When an ECG signal is received, the noise level is first estimated. Then, based on the estimated noise level and our proposed fuzzy rules, different multiwavelets, pre- and post-filters are integrated together. A hard thresholding is applied on the multiwavelet coefficients. According to extensive numerical computer simulations, our proposed fuzzy rule based multiwavelet denoising algorithm outperforms traditional multiwavelet denoising algorithms by $30 \%$.
\end{abstract}

\section{INTRODUCTION}

$\mathrm{D}$ ENOISING is to reduce noise levels while the signal degradation is minimized. As noise is found in everywhere and usually corrupted with signals, denoising is important and useful in many engineering applications.

Among the existing denoising algorithms, wavelet denoising algorithms (Thresholding is applied on the wavelet coefficients.) are the most common approaches for denoising because wavelets exploit both the time and the frequency domain information of signals and hence wavelet denoising approaches can achieve good performances. In the past, many researches studied the effects of various thresholding functions on the denoising performances. Nowadays, soft thresholding wavelet denoising methods [8, 9] are well understood and widely applied to images and ECG signals. Further extension from soft thresholding wavelet denoising approaches to a fuzzy thresholding wavelet denoising approach [7] was also proposed.

Although scalar wavelets are widely employed for signal processing, it is difficult for scalar wavelets to achieve orthogonality, symmetry, short support and high vanishing moments simultaneously. Multiwavelets were proposed recently $[1,2,3]$, and it was found that multiwavelets could achieve all the above four properties simultaneously. Owing to these advantages, various thresholding functions for multiwavelet denoising approaches were proposed [4, 5, 6].

Although multiwavelet denoising techniques have already been proved to be efficient for signal denoising, the effects of various multiwavelets, pre- and post-filters on denoising performances are unknown. Hence, methods for selecting multiwavelets, pre- and post-filters are unknown. The aim of this paper is to suggest a method to integrate different multiwavelets, pre- and post-filters together to obtain suboptimal results for ECG signal denoising. Since two types of Chui Lian (CL) multiwavelets [2, p.289, p.295] and Qingtnag Jiang (QJ) multiwavelet [3] are the most common multiwavelets used for signal processing, these three multiwavelets are chosen for the investigation. Besides, thirty five commonly used pre- and post-filters are also selected for the investigation. In this paper, ECG signals are assumed to be corrupted by additive white Gaussian noises (AWGN). A hard thresholding is applied on the multiwavelet coefficients. The fuzzy rules are established based on the following methods: A set of input output pairs of the denoising performances using different multiwavelets, pre- and post-filters at different noise levels are obtained, where the denoising performances are calculated based on the mean square error (MSE) criterion. We fixed a number of fuzzy rules, and design the fuzzy membership functions based on the input output pairs obtained from the numerical computer simulations. Once the fuzzy rules are designed, a new ECG signal can be denoised based on these fuzzy rules.

The outline of this paper is as follows: Section II and Section III will review some basic multiwavelet theories and fuzzy mechanisms, respectively. Our proposed fuzzy rule based denoising algorithm is discussed in Section IV. Numerical computer simulation results are shown in Section V. Finally a conclusion is drawn in Section VI.

\section{MultiwaVELET THEORIES}

A multiresolution analysis of $L^{2}(\Re)$ generated by several scaling functions $\phi^{1}, \phi^{2}, \cdots, \phi^{r}$ can be expressed as an increasing sequence of function subspaces $V_{j}$ in $L^{2}(\Re)$ for $j \in Z$ as follows:

$$
\{0\} \subset \cdots \subset V_{-1} \subset V_{0} \subset V_{1} \cdots \subset L^{2}(\Re) .
$$

Subspaces $V_{j}$ are generated by a set of scaling function $\left\{\phi^{1}, \phi^{2}, \cdots, \phi^{r}\right\}$ such that

$$
V_{j} \equiv \operatorname{clos}_{L^{2}(\Re)}\left\langle\phi_{j, k}^{m}: 1 \leq m \leq r, k \in Z\right\rangle \quad \forall j \in Z .
$$


In other words, $V_{j}$ is the closure of the linear span of $\left\{\phi_{j, k}^{m}\right\}_{1 \leq m \leq r, k \in Z}$ in $L^{2}(\Re)$, where

$$
\phi_{j, k}^{m}(x) \equiv 2^{j / 2} \phi^{m}\left(2^{j} x-k\right) \forall x \in \mathfrak{R} .
$$

A sequence of multiresolution subspaces $V_{j}$ generated by a set of multiscaling functions $\left\{\phi^{1}, \phi^{2}, \cdots, \phi^{r}\right\}$ gets finer and finer as $j$ increases.

The interspaces $W_{j} \subset L^{2}(\Re)$ is defined such that $V_{j+1} \equiv V_{j} \oplus W_{j} \quad \forall j \in Z$, where the plus sign with a circle denotes a nonorthogonal direct sum. $W_{j}$ is the complement to $V_{j}$ in $V_{j+1}$, and thus $W_{j}$ and $W_{l}$ with $j \neq l$ are disjoint but may not be orthogonal to each other. If $W_{j} \perp W_{l} \quad \forall j \neq l$, this corresponds to semiorthogonal wavelet spaces. By the nature of construction, subspaces $W_{j}$ can be generated by $r$ base functions $\psi^{1}, \psi^{2}, \cdots, \psi^{r}$ that are known as multiwavelets. The subspace $W_{j}$ is the closure of the linear span of $\left\{\psi_{j, k}^{m}\right\}_{1 \leq m \leq r, k \in Z}$ in $L^{2}(\mathfrak{R})$ such that

$$
W_{j} \equiv \operatorname{clos}_{L^{2}(\Re)}\left\langle\psi_{j, k}^{m}: 1 \leq m \leq r, k \in Z\right\rangle \quad \forall j \in Z
$$

where

$$
\psi_{j, k}^{m}(x) \equiv 2^{j / 2} \psi^{m}\left(2^{j} x-k\right) \forall x \in \mathfrak{R}
$$

The multiscaling functions and multiwavelets can be expressed as vector functions as follows:

$$
\phi(x) \equiv\left(\begin{array}{c}
\phi^{1}(x) \\
\phi^{2}(x) \\
\vdots \\
\phi^{r}(x)
\end{array}\right) \text { and } \psi(x) \equiv\left(\begin{array}{c}
\psi^{1}(x) \\
\psi^{2}(x) \\
\vdots \\
\psi^{r}(x)
\end{array}\right) \forall x \in \mathfrak{R}
$$

Also, in vector form, we have:

$$
\boldsymbol{\phi}_{j, k}(x) \equiv 2^{j / 2} \boldsymbol{\phi}\left(2^{j} x-k\right) \text { and } \boldsymbol{\psi}_{j, k}(x) \equiv 2^{j / 2} \boldsymbol{\psi}\left(2^{j} x-k\right) \forall x \in \mathfrak{R} \text {. }
$$

Since the multiscaling functions $\phi^{m} \in V_{0}$ and the multiwavelets $\psi^{m} \in W_{0}$ are all in $V_{1}$, and since $V_{1}$ is generated by $\left\{\phi_{1, k}^{m}(x)=2^{j / 2} \phi^{m}(2 x-k)\right\}_{1 \leq m \leq r, k \in Z}$, there exists two $\ell^{2}$ matrix sequences $\mathbf{H}_{n}$ and $\mathbf{G}_{n}$ for $n \in Z$ such that a two scale relation for the multiscaling function $\phi(x)$ can be defined as:

$$
\phi(x) \equiv 2 \sum_{n \in Z} \mathbf{H}_{n} \phi(2 x-n) \quad \forall x \in \mathfrak{R}
$$

which is also called as a two scale matrix refinement equation, and for multiwavelet $\psi(x)$, we have:

$$
\boldsymbol{\psi}(x) \equiv 2 \sum_{n \in Z} \mathbf{G}_{n} \boldsymbol{\psi}(2 x-n) \quad \forall x \in \mathfrak{R},
$$

where $\mathbf{H}_{n}$ and $\mathbf{G}_{n}$ are $r \times r$ square matrices. In the implementation, the multiwavelet is implemented via prefiltering the input signal, passing the pre-filtered signal to a vector valued filter bank with the impulse response of the analysis and the synthesis filters being characterized by $\mathbf{H}_{n}$ and $\mathbf{G}_{n}$, respectively, and then post-filtering the signal to reconstruct the original signal.

\section{FUZZY MECHANISMS}

Fuzzy logic is a mathematical formalism that attempts to imitate the way humans make decisions. Through the concept of the fuzzy grade membership, fuzzy set theory and fuzzy logic allow a simple mathematical expression to capture expert knowledge to a system [11], [12].

In our fuzzy system, all fuzzy membership functions are defined as trapezoidal fuzzy membership functions. Trapezoidal fuzzy membership functions are convex, normal, compact, and easy to implement. An example of a trapezoidal fuzzy membership function is shown in Figure 1 .

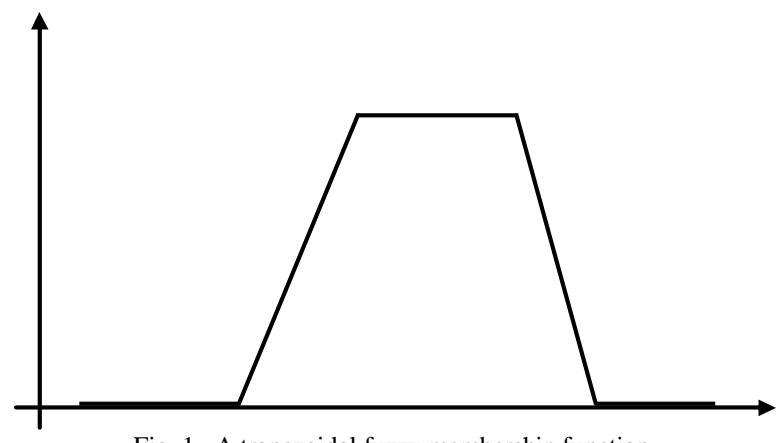

Fig. 1. A trapezoidal fuzzy membership function.

Denote $n$ as the dimension of the input vector, $A_{1}^{j}, A_{2}^{j}, \ldots, A_{k_{j}}^{j}$ for $j=1, \cdots, n$ as the fuzzy sets for the input $x_{j}$, and $B_{1}, B_{2}, \ldots, B_{m}$ as the fuzzy sets for the output $z$. The system comprises a set of IF THEN fuzzy rules having the following form:

$$
\begin{gathered}
\text { IF } x_{1} \text { is } A_{\theta_{1}}^{1} \text { AND } x_{2} \text { is } A_{\theta_{2}}^{2} \text { AND...AND } x_{n} \text { is } A_{\theta_{n}}^{n} . \\
\text { THEN } z \text { is } R_{\theta_{1}, \theta_{2}, \ldots, \theta_{n}}
\end{gathered}
$$

where $1 \leq \theta_{j} \leq k_{j}$ and $R_{\theta_{1}, \theta_{2}, \ldots, \theta_{n}} \in\left\{B_{1}, B_{2}, \ldots, B_{m}\right\}$. Actually, the input space of $x_{j}$ is partitioned into fuzzy sets 
$A_{1}^{j}, A_{2}^{j}, \ldots, A_{k_{j}}^{j}$ for $j=1, \cdots, n$, and the output space of $z$ is partitioned into fuzzy sets $B_{1}, B_{2}, \ldots, B_{m}$.

The trapezoidal fuzzy membership function $\mu(x)$ in Figure 1 is defined as follows:

$$
\mu(x)=\left\{\begin{array}{cc}
0, & \text { if } x \in(-\infty, a) \cup(d, \infty), \\
\frac{x-a}{b-a}, & \text { if } \in[a, b], \\
\frac{x-d}{c-d}, & \text { if } x \in[c, d], \\
1, & \text { if } x \in(b, c),
\end{array}\right.
$$

where $a<b<c<d \in \mathfrak{R}$.

\section{Our Proposed FuZZy Multiwavelet DenOIsing SYSTEM}

Our proposed fuzzy multiwavelet denoising system comprises with six blocks as shown in Figure 2. The ECG signals used in the investigation are from the Beth Israel Deaconess Medical Center at Boston [10]. They are contaminated by additive white Gaussian noises with zero mean and variances $\sigma^{2}$, which can be estimated using existing adaptive signal processing techniques.

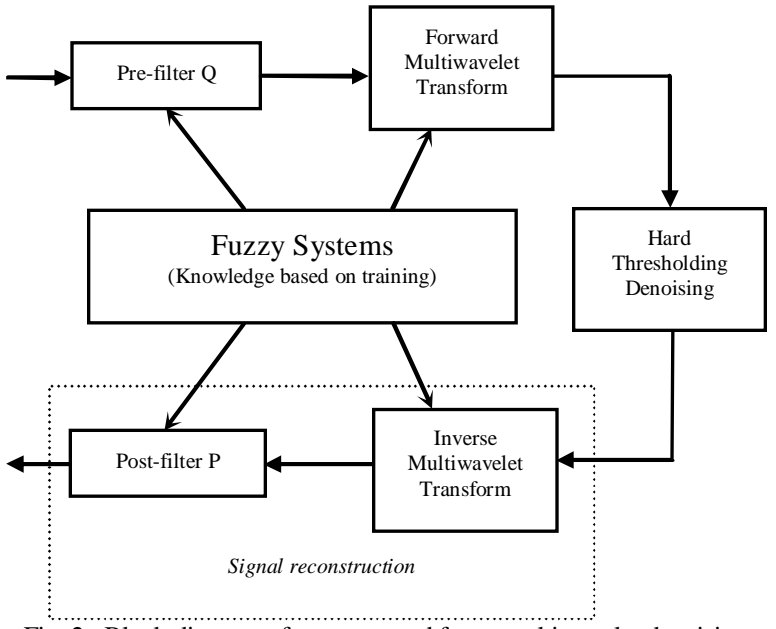

Fig. 2. Block diagram of our proposed fuzzy multiwavelet denoising system.

The original signal $s_{k}$ is first added with noise. Then the signal is pre-filtered by a filter with filter response $Q$. After that, a multiwavelet transform is applied. The pre-filter is used to map the data $s_{k}$ into multiple streams because multiwavelet transform requires the input data having multiple streams. A threshold level $t_{n}$ developed by Donoho [9] as follows

$$
t_{n}=\sigma \sqrt{2 \log n}
$$

is employed. The denoised signal $\hat{s}_{i}$ is then inverse multiwavelet transformed. The final block of data is then post-filtered by a filter with the filter response $P$ to obtain a denoised signal. The set of pre-filters and post-filters has to satisfy $P Q=I$, where $I$ is the identity filter response, that is:

$$
I=\left[\begin{array}{ll}
1 & 0 \\
0 & 1
\end{array}\right] \text {. }
$$

In order to measure the denoising performance, MSE is used as the criterion, that is

$$
\operatorname{MSE}=\frac{1}{N} \sum_{n=0}^{N-1}[s(n)-\hat{s}(n)]^{2},
$$

where $N$ is the total signal length.

The fuzzy systems determine the best combination of multiwavelets, pre- and post-filters for minimizing the MSE of the reconstructed signal. The knowledge on the effects of employing different multiwavelets, pre- and post-filters at different noise levels on denoising performances is obtained based on training which is discussed in the next section.

\section{NuMERICAL COMPUTER SimUlation RESUlTS}

In order to demonstrate the effects of employing different multiwavelets, pre- and post-filters on the denoising performances, numerical computer simulations were performed to recover a signal with 1000 samples embedded in the zero mean additive white Gaussian noises with various noise powers. The original ECG signal is shown in Figure 3.

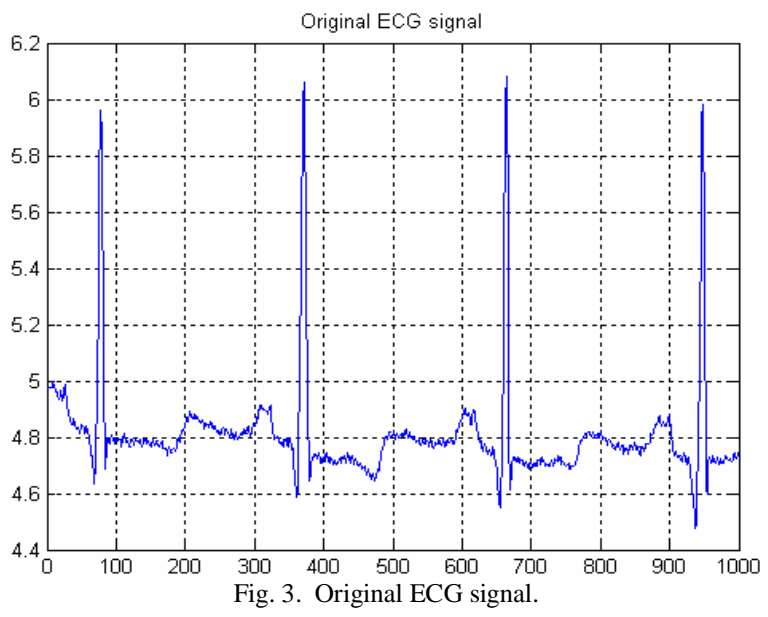

By employing different combinations of the three multiwavelets and the thirty five pre- and post-filters listed in Table 1, the percentage improvements of the MSE of the reconstructed signals for each combination are obtained. Table 2 lists the multiwavelet, pre- and post-filters that give the best denoising performances when the ECG signal is corrupted at different noise powers. Figure 4 and Figure 5 show an example of a noisy ECG signal with $\sigma$ equal to 
0.070 and the corresponding denoised signal, respectively. It can be seen from the figures that the noise level of the reconstructed signal is significantly reduced.

TABLE I

MULTIWAVELETS, PRE- AND POST- FILTERS

\begin{tabular}{|c|c|c|}
\hline & \multicolumn{2}{|c|}{ Name } \\
\hline \multirow{2}{*}{$\begin{array}{l}\text { Multiwavelet } \\
\text { Kernels }\end{array}$} & \multicolumn{2}{|c|}{ Chiu Lian $[2$, p.289, p.295] } \\
\hline & \multicolumn{2}{|c|}{ Qingtang Jiang [3] } \\
\hline \multirow[b]{4}{*}{$\begin{array}{l}\text { Pre- and post- } \\
\text { filters }\end{array}$} & Symlets & 2 to 8 \\
\hline & Coiflets & 1 to 5 \\
\hline & Daubenchies & 1 to 8 \\
\hline & Biorthogonal & 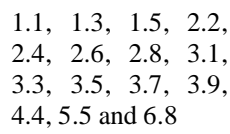 \\
\hline
\end{tabular}

TABLE II

SETS OF FUZZY RULES FOR DENOISING ECG SIGNALS.

\begin{tabular}{|l|l|}
\hline Rules & Kernels \\
\hline IF $0.030 \leq \sigma \leq 0.058$ then use & CL2 or QJ \\
\hline IF $0.052 \leq \sigma \leq 0.076$ then use & CL2 \\
\hline IF $0.069 \leq \sigma \leq 0.100$ then use & QJ \\
\hline Rules & Filters \\
\hline IF $0.030 \leq \sigma \leq 0.040$ then use & Symlets 2 \\
\hline IF $0.032 \leq \sigma \leq 0.080$ then use & Biorthogonal 3.3 \\
\hline IF $0.074 \leq \sigma \leq 0.100$ then use & Biorthogonal 3.1 \\
\hline
\end{tabular}

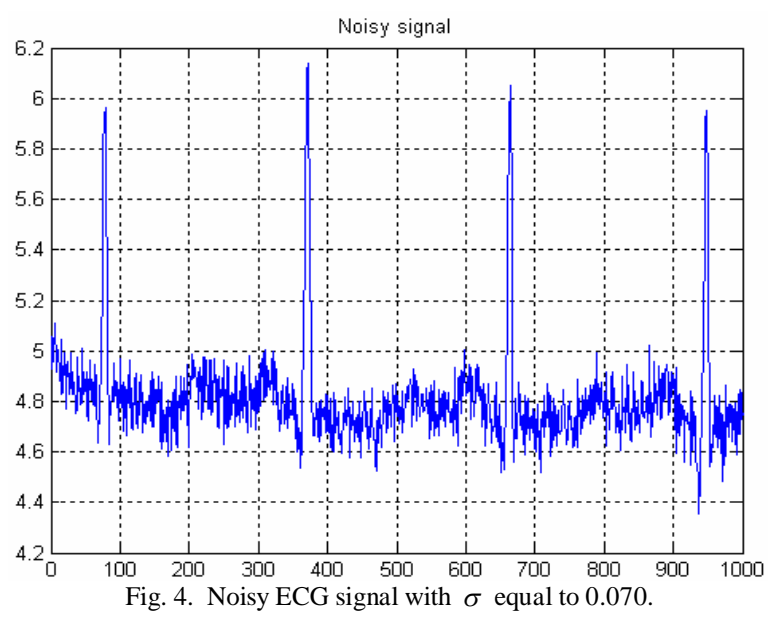

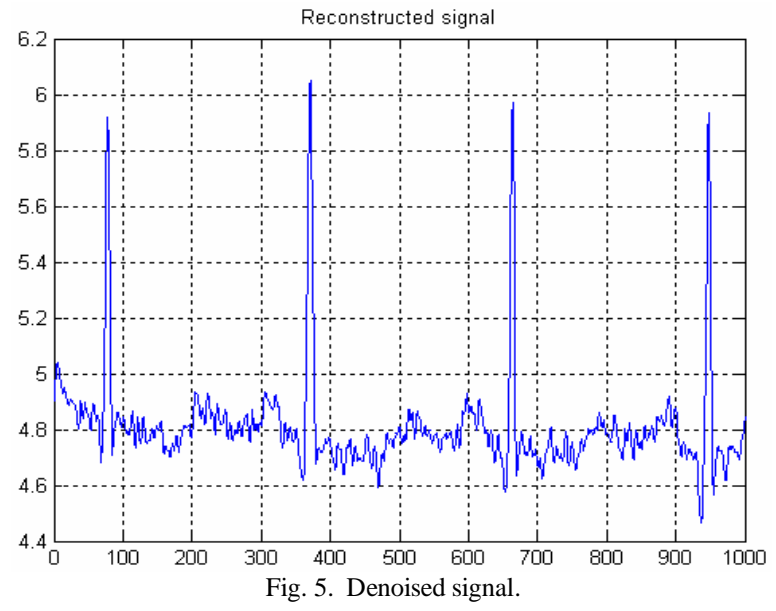

The fuzzy variable $\sigma$ is described by six linguistic terms with trapezoidal fuzzy membership functions as shown in Figure 6 and Figure 7. The linguistic terms divide each partition of multiwavelets and pre- and post- filters into three segments. The segments for multiwavelets are Kernel Low (KL), Kernel Medium (KM) and Kernel Big (KB), while that for the pre- and post-filters are Filter Low (FL), Filter Medium (FM) and Filter Big (FB).
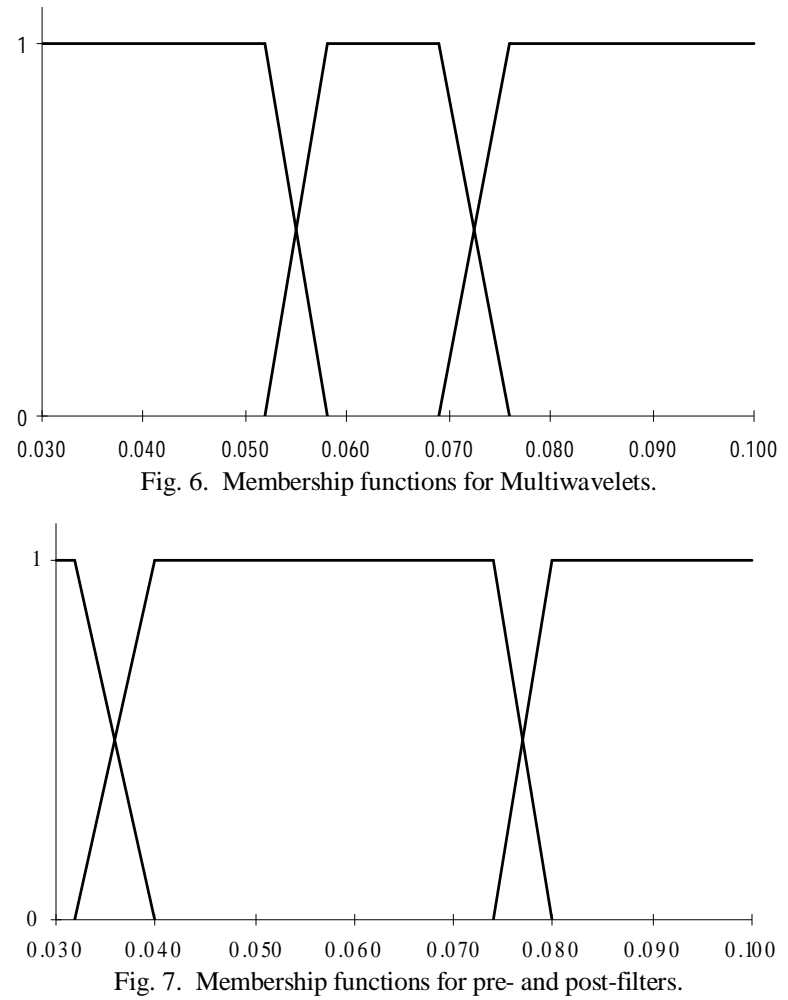

Figure 8 shows the average percentage improvement of the MSE of the denoising signals using the fuzzy and the nonfuzzy approaches. The nonfuzzy approach is performed by applying the univariate soft thresholding to GHM multiwavelet coefficients [5]. Comparing the results of these two methods, the fuzzy approach outperforms the nonfuzzy approach by more than $20 \%$ for all noise powers. 


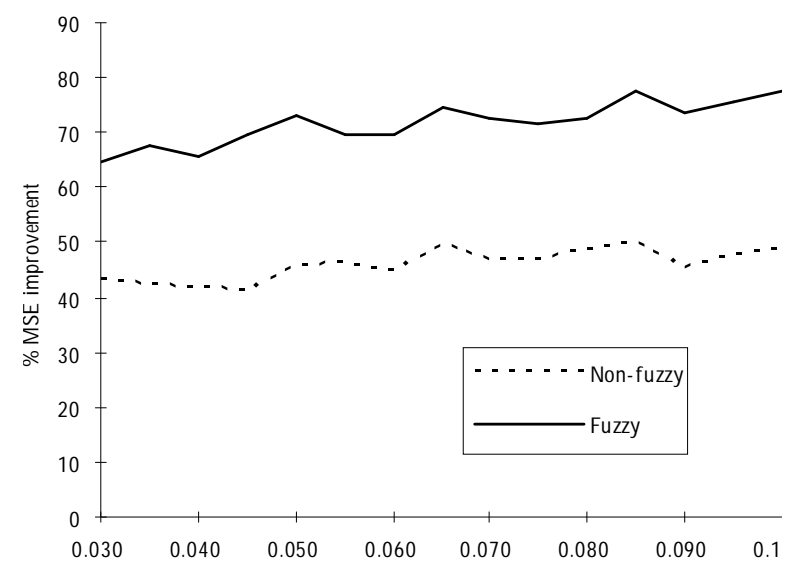

Fig. 8. Comparison on the denoising performances.

\section{CONCLUSIONS}

This paper proposes a fuzzy approach for multiwavelet denoising of ECG signals. The method integrates various multiwavelets, pre- and post-filters together so that the denoising performance is significantly improved.

\section{ACKNOWLEDGMENT}

The work obtained in this paper was supported by a research grant from Queen Mary College, University of London.

\section{REFERENCES}

[1] V.Strela, P. N. Heller, G. Strang, P. Topiwala, and C.Heil, "The application of multiwavelet filter banks to image processing," IEEE Transactions on image processing, vol. 8, no.4, pp.548-563, April 1999.

[2] C. K. Chui and J. A. Lian, "A study of orthonormal multi-wavelets," Applied Numerical Mathematics, vol. 20, pp. 273-298, 1996.

[3] Qingtang Jiang, "On the design of multifilter banks and orthonormal multiwavelets bases," IEEE Transactions on signal processing, vol. 46, no. 12, pp. 3292-3303, December 1998.

[4] T. R. Downie and B. W. Silerman, "The discrete multiple wavelet transform and thresholding methods," IEEE Transactions on signal processing, vol. 46, no. 9, pp. 2558-2561, September 1998.

[5] T. D. Bui and G. Chen, "Translation-invariant denoising using multiwavelets," IEEE Transactions on signal processing, vol. 40, no. 12, pp. 3414-3420, December 1998.

[6] M. Jansen and A. Bultheel, "Multiple wavelet threshold estimation by generalized cross validation for images with correlated noise," IEEE Transactions on image processing, vol. 8, no. 7, pp. 947-953, July 1999.

[7] L.K. Shark and C.Yu, "Denoising by optimal fuzzy thresholding in wavelet domain," Electronics Letters, vol. 36, no. 6, 16th March 2000.

[8] P. M. Agante, J. P. Marques de Sa, "ECG noise filtering using wavelets with soft-thresholding methods," Computers in Cardiology, vol. 26, pp. 535-538, 1999.

[9] D. L. Donoho, "De-noising by soft-thresholding," IEEE Transactions on information theory, vol. 41, no. 3, pp. 613-627, May 1995.

[10] http://www.physionet.org/physiobank/database/mitdb/

[11] D. Ruan and E. E. Kerre, Fuzzy if-then rules in computational intelligence: theory and applications, Kluwer Academic, 2000.

[12] P. P. Wang, Da Ruan and E. E. Kerre, Fuzzy logic: a spectrum of theoretical and practical issues (studies in fuzziness and soft computing), Springer, 2007. 